\title{
K-shell transitions in L-shell ions with the EBIT calorimeter spectrometer
}

\author{
Natalie Hell ${ }^{1,2}$, Greg V. Brown ${ }^{2}$, Jörn Wilms ${ }^{1}$, Peter Beiersdorfer ${ }^{2}$, \\ Richard L. Kelley ${ }^{3}$, Caroline A. Kilbourne ${ }^{3}$ and F. Scott Porter ${ }^{3}$ \\ ${ }^{1}$ Dr. Remeis-Sternwarte \& ECAP, Friedrich-Alexander Universität Erlangen-Nürnberg, \\ Sternwartstr. 7, 96049 Bamberg, Germany \\ email natalie.hell@sternwarte.uni-erlangen.de \\ ${ }^{2}$ Lawrence Livermore National Laboratory, 7000 East Ave., Livermore CA 94550, USA \\ ${ }^{3}$ NASA-GSFC, 8800 Greenbelt Road, Greenbelt, MD 20771, USA
}

\begin{abstract}
With the large improvement in effective area of Astro-H's micro-calorimeter soft X-ray spectrometer (SXS) over grating spectrometers, high-resolution X-ray spectroscopy with good signal to noise will become more commonly available, also for faint and extended sources. This will result in a range of spectral lines being resolved for the first time in celestial sources, especially in the Fe region. However, a large number of X-ray line energies in the atomic databases are known to a lesser accuracy than that expected for Astro-H/SXS, or have no known uncertainty at all. To benchmark the available calculations, we have therefore started to measure reference energies of $\mathrm{K}$-shell transition in L-shell ions for astrophysically relevant elements in the range $11 \leqslant Z \leqslant 28$ ( $\mathrm{Na}$ to $\mathrm{Ni}$ ), using the Lawrence Livermore National Laboratory's EBIT-I electron beam ion trap coupled with the NASA/GSFC EBIT calorimeter spectrometer (ECS). The ECS has a resolution of $\sim 5 \mathrm{eV}$, i.e., similar to Astro-H/SXS and Chandra/HETG. A comparison to crystal spectra of lower charge states of sulfur with $\sim 0.6 \mathrm{eV}$ resolution shows that the analysis of spectra taken at ECS resolution allows to determine the transition energies of the strongest components.
\end{abstract}

Keywords. atomic data; X-rays: general; methods: laboratory

\section{Introduction}

The microcalorimeter soft X-ray spectrometer (SXS) onboard the upcoming Astro-H mission (Takahashi et al. 2010) will bring a large improvement in effective area over the currently available grating spectrometers on the XMM-Newton and Chandra X-ray observatories. This will make high-resolution spectroscopy more commonly available and extend its application to faint and extended sources. Consequently, a plethora of new spectral features is expected to be observed with the new instrument. For example, in a variety of brighter sources - including X-ray binaries, AGN and solar flares - Kshell transitions of L-shell ions of Si and $\mathrm{S}$ have already been observed with grating spectrometers. With the larger effective area, similar features should become apparent also for other elements and additional sources. Detailed analysis of the Fe $\mathrm{K}$ lines in CCD spectra of supernova remnants reveals a complex ionization structure (Yamaguchi et al. 2014) and some SNRs show signatures of the trace elements Cr and Mn (Hughes et al. 2014). Astro-H SXS will not only be able to resolve the charge balance for these Fe group elements; it will also detect and resolve weak K-shell lines from lower odd-Z trace elements that are more abundant than $\mathrm{Cr}$ and $\mathrm{Mn}$, but generally too close in energy to stronger lines for CCD resolution (Hughes et al. 2014). To take full advantage of the diagnostic capabilities of these lines, accurate atomic reference data are crucial, starting with transition energies. However, inner-shell transitions are not commonly available in 


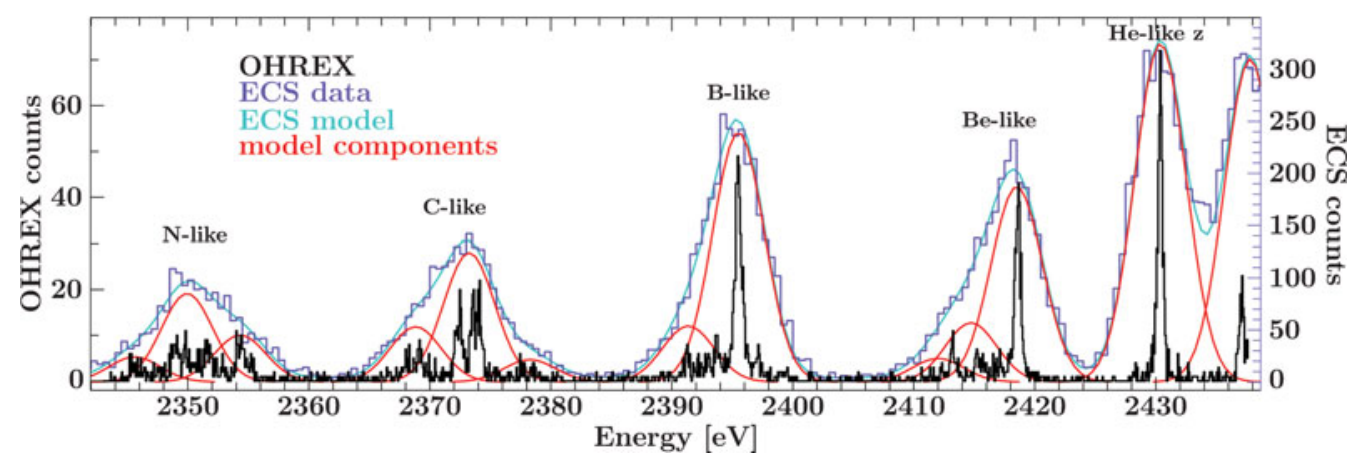

Figure 1. Crystal spectra of sulfur in comparison with ECS spectra and its fit model.

current databases, and although some calculations do exist, their accuracy is unknown. To benchmark the theoretical reference energies, we are measuring K-shell transitions in L-shell ions of astrophysically relevant ions, described here on the example of Si and S.

\section{Measurements}

We use the Lawrence Livermore electron beam ion trap EBIT-I (Marrs et al. 1988), where the electron beam ionizes and excites injected neutral material. The ions are trapped by the potential well created through a set of three drift tubes. Employing short cycles of dumping and refilling the trap together with a large supply of neutrals from the gas injector, allows us to create a low charge balance. We record the photons emitted from the trapped ions with the EBIT calorimeter spectrometer (ECS; Porter et al. 2008). The ECS is similar to the calorimeter on-board Astro- $\mathrm{H}$ and has a resolution of about $5 \mathrm{eV}$. At this resolution, the principle lines of individual L-shell ions are clearly separated (Fig. 1). The line profiles and the near constant energy resolution allow us to further disentangle the strongest transitions in these line blends. The energy scale is calibrated using well-known reference lines like the Rydberg series of He- and H-like ions. The accuracy of the measured strong lines in $\mathrm{Si}$ and $\mathrm{S}$ is $\lesssim 0.5 \mathrm{eV}$, corresponding to a Doppler shift of $90 \mathrm{~km} \mathrm{~s}^{-1}$, i.e., slightly better than the calibration of Chandra HETG. For the S spectra, a comparison of the model components of the fit to the ECS data with a higher resolution $(\sim 0.6 \mathrm{eV}$ FWHM) spherical crystal spectrometer, dubbed OHREX, shows that the strong transitions are correctly reproduced by our analysis of the ECS data (Fig. 1). The ECS measurements are available for a few of the astrophysically relevant elements between $Z=11$ and $Z=28$, including Si and S (Hell et al. 2013), and we are continuing the effort to fill in the gaps.

This work was supported by LLNL under Contract DE-AC52-07NA27344, by NASA grants to LLNL and NASA/GSFC, and by the European Space Agency under ESA contract No. 4000114313/15/NL/CB.

\section{References}

Hell, N., Miškovičová, I., Brown, G. V., et al. 2013, Phys. Scr., T156, 014008

Hughes, J. P., Safi-Harb, S., Bamba, A., et al. 2014, arxiv:1412.1169v1

Marrs, R. E., Levine, M. A., Knapp, D. A., \& Henderson, J. R. 1988, Phys. Rev. Lett., 60, 1715

Porter, F. S., Beiersdorfer, P., Brown, G. V., et al. 2008, J. Low Temp. Phys., 151, 1061

Takahashi, T., Mitsuda, K., Kelley, R., et al. 2010, in: M. Arnaud, S. S. Murray, T. Takahashi (eds.), Space Telescopes and Instrumentation 2010: UV to Gamma Ray, Proc. SPIE 7732

Yamaguchi, H., Eriksen, K. A., Badenes, C., et al., 2014, ApJ 780, 136 